\title{
Femoral artery cannulation as a safe alternative for aortic dissection arch repair in the era of axillary artery cannulation
}

\author{
Guang Tong ${ }^{1 \#}$, Dong-Lin Zhuang ${ }^{1,2 \#}$, Zhong-Chan Sun ${ }^{3}$, Ze-Rui Chen ${ }^{1}$, Rui-Xin Fan ${ }^{1}$, Tu-Cheng Sun ${ }^{1}$ \\ ${ }^{1}$ Department of Cardiac Surgery, Guangdong Cardiovascular Institute, Guangdong Provincial Key Laboratory of South China Structural Heart \\ Disease, Guangdong Provincial People's Hospital, Guangdong Academy of Medical Sciences, Guangzhou, China; ${ }^{2}$ Shantou University Medical \\ College, Shantou, China; ${ }^{3}$ Department of Cardiology, Guangdong Cardiovascular Institute, Guangdong Provincial Key Laboratory of South China \\ Structural Heart Disease, Guangdong Provincial People's Hospital, Guangdong Academy of Medical Sciences, Guangzhou, China \\ Contributions: (I) Conception and design: G Tong, DL Zhuang, ZC Sun, TC Sun; (II) Administrative support: RX Fan; (III) Provision of \\ study materials or patients: G Tong, DL Zhuang, ZC Chen; (IV) Collection and assembly of data: G Tong, DL Zhuang; (V) Data analysis and \\ interpretation: G Tong, DL Zhuang, ZC Sun; (VI) Manuscript writing: All authors; (VII) Final approval of manuscript: All authors. \\ "These authors contributed equally to this work. \\ Correspondence to: Rui-Xin Fan, Tu-Cheng Sun. Department of Cardiac Surgery, Guangdong Cardiovascular Institute, Guangdong Provincial Key \\ Laboratory of South China Structural Heart Disease, Guangdong Provincial People's Hospital, Guangdong Academy of Medical Sciences, Yindong \\ Building, No. 96 Dongchuan Road, Yuexiu District, Guangzhou 510080, China. Email: syfanruixin@sina.com; suntucheng@126.com.
}

Background: To evaluate the safety and efficacy of femoral artery cannulation as an alternative to axillary artery cannulation, we retrospectively compared outcomes between patients with axillary or femoral artery cannulation during open aortic arch repair for type A aortic dissection (TAAD).

Methods: Between January 2014 and January 2019, 646 patients underwent open aortic arch repair with circulatory arrest for TAAD using antegrade selective cerebral perfusion (SACP) and were divided into two groups according to the site of arterial cannulation: an axillary artery group (axillary group, $\mathrm{n}=558$ ) or a femoral artery group (femoral group, $n=88$ ). The axillary artery was considered as the primary cannulation site, and the femoral artery was used as an alternative when axillary artery cannulation was deemed unsuitable or had failed. Propensity score matching was performed to correct baseline differences.

Results: After propensity score matching, the patients' characteristics were comparable between groups ( $\mathrm{n}=85$ in each). The incidence of in-hospital mortality $(10.6 \%$ vs. $14.1 \% ; \mathrm{P}=0.642)$ and stroke $(3.5 \%$ vs. $5.9 \% ; \mathrm{P}=0.720)$ were comparable between the axillary and femoral groups. The incidence of newly required dialysis was lower in the femoral group, but the difference was not statistically significant (34.1\% vs. 20.0\%; $\mathrm{P}=0.050)$. Other outcomes and major adverse events were comparable.

Conclusions: Femoral artery cannulation produced similar perioperative outcomes to axillary cannulation after open arch repair for TAAD. The femoral artery can be used as a safe and effective alternative to the axillary artery for arterial cannulation in TAAD patients undergoing open arch repair.

Keywords: Aortic arch; femoral artery cannulation; type A aortic dissection (TAAD)

Submitted Jun 02, 2020. Accepted for publication Nov 18, 2020.

doi: $10.21037 /$ jtd-20-2113

View this article at: http://dx.doi.org/10.21037/jtd-20-2113

\footnotetext{
$\wedge$ ORCID: 0000-0002-9367-7891.
} 


\section{Introduction}

Selecting the ideal arterial cannulation site for Stanford type A aortic dissection (TAAD) patients undergoing aortic arch repair is critical for optimal outcomes. Growing evidence supports the superiority of axillary artery cannulation because it preserves antegrade flow in the descending aorta, thereby reducing the risk for embolization, and facilitates the administration of selective antegrade cerebral perfusion (ASCP) during aortic arch repair (1-3). The axillary artery is recommended as the first choice for cannulation by the 2014 European Society of Cardiology Guidelines on the Diagnosis and Treatment of Aortic Diseases (class of recommendation I, level of evidence C) (4).

However, the cannulation strategy should be individualized in TAAD repair because the extent of dissection and vessel anatomy vary among patients (5). Technical problems and complications may also occur during axillary cannulation (6), so an alternative cannulation site should always be included in the surgical strategy.

The femoral artery was the most commonly used arterial cannulation site in arch repair for TAAD before the introduction of axillary cannulation $(7,8)$. Despite concerns that reversed flow in the thoracoabdominal aorta may increase the risk of retrograde brain embolization, dissection, and organ malperfusion (9), femoral artery cannulation is still widely used by many institutes with good outcomes $(10,11)$.

An individualized strategy is used at the Guangdong Cardiovascular Institute. The right axillary artery is the first choice for cannulation in the majority of patients, and the femoral artery is the alternative route in patients for whom axillary artery cannulation is considered unsuitable or has failed.

We hypothesized that femoral artery cannulation produces comparable outcomes to axillary cannulation when used as an alternative, so we retrospectively compared the outcomes between TAAD patients undergoing open aortic arch repair with the axillary or femoral artery used as the primary arterial cannulation site. We present our study in accordance with the STROBE reporting checklist (available at http://dx.doi.org/10.21037/jtd-20-2113).

\section{Methods}

\section{Study population}

Between January 2014 and January 2019, 943 TAAD patients underwent open aortic arch repair with circulatory arrest at the Guangdong Cardiovascular Institute. After excluding patients who underwent two arterial cannulations $(n=293)$ or ascending aorta cannulation $(n=4)$ at the start of cardiopulmonary bypass (CPB), the remaining 646 patients were divided into two groups according to the site of arterial cannulation: an axillary group $(\mathrm{n}=558)$ or a femoral group $(\mathrm{n}=88)$. Medical records were reviewed. In-hospital mortality, stroke, and early complications including re-exploration for bleeding, extracorporeal membrane oxygenation (ECMO) use, mediastinitis, tracheostomy, and renal failure requiring dialysis were compared between groups both before and after propensity score matching. The study protocol was approved by the Ethics Committee of Guangdong Provincial People's Hospital [approval number: No. GDREC2018322H(R2)]. Owing to the retrospective nature of the study, individual consent was waived.

\section{Surgical technique}

\section{Selection of cannulation site}

All procedures were performed by two experienced surgeons (R Fan, T Sun). For most patients, the primary cannulation site was determined preoperatively after carefully reviewing the patient's condition and computed tomography (CT) imaging. Factors including blood pressure in all four limbs, arch vessel anatomy, dissection involvement, true lumen diameters, and false-lumen thrombus formation were taken into consideration. The right axillary artery was the first choice for arterial cannulation, with the femoral artery as the secondary cannulation site. After dissection to the artery, the artery was palpated and carefully examined. Before cannulation, the artery was controlled both proximally and distally with all branches snared. The artery was half transected for inspection of the vessel lumen to avoid inadvertent entry to the false lumen. After cannulation, blood return in the cannula and pump resistance were carefully assessed before CPB initiation. Of 88 patients undergoing femoral cannulation, 47 had extensive axillary arterial dissection involvement or possible intimal tear within the innominate or subclavian artery upon preoperative CT review; 29 patients were converted to femoral artery cannulation due to insufficient vessel caliber upon surgical inspection, low blood return, or elevated pump resistance; and 12 patients had an aberrant right subclavian artery (ARSA) originating from the descending aorta. 


\section{Assessment of perfusion}

Perfusion of vital organs was assessed throughout the procedure. Body temperature monitoring was achieved through the bladder and nasal cavity. Coronary perfusion was assessed with transesophageal echocardiography (TEE) with the blood flow in the left coronary ostia and regional wall motion in the left ventricle being examined. Cerebral perfusion was monitored with regional cerebral oxygen saturation $\left(\mathrm{rSO}_{2}\right)$ in the bilateral frontal lobes using nearinfrared spectroscopy. Perfusion in the lower extremities was assessed by inspection and blood pressure monitoring of the dorsalis pedis artery or femoral artery.

\section{Technique for cerebral perfusion}

ASCP in combination with moderate hypothermia (nasopharyngeal temperature $20-24^{\circ} \mathrm{C}$ ) is our standard neurocerebral protection. For patients in the axillary group, unilateral hemisphere perfusion was performed via right axillary artery cannulation during systemic circulatory arrest. For patients in the femoral artery group, unilateral hemisphere perfusion was performed via direct cannulation of the right or left common carotid artery. A cerebral flow rate of $10-15 \mathrm{~mL} / \mathrm{kg} / \mathrm{min}$ was adjusted to maintain a radial arterial pressure between 40 and $70 \mathrm{mmHg}$.

\section{Statistical analysis}

Continuous variables are expressed as mean \pm standard deviation (SD). Categorical variables are presented as percentages. Student's $t$-test or Mann-Whitney test was used for continuous variables. Pearson's $\chi^{2}$ or Fisher's exact test was used for categorical variables.

To minimize the preoperative differences between the axillary and femoral groups, a propensity score match analysis was performed based only on preoperative characteristics and using a 1:1 greedy match algorithm without replacement. Axillary versus femoral cannulation was the dependent variable in the logistic regression model used to compute the propensity scores, and the following preoperative variables were included: connective tissue disease, cerebral malperfusion, unstable status, history of stroke, renal insufficiency, hypertension, history of heart/ aortic surgery, and renal malperfusion.

The matching process yielded 85 matched pairs. After matching, we assessed the balance within the matched pairs using the standardized differences in covariate means (12). All of the standardized differences were less than $10 \%$, except for age, smoking, coronary heart disease, and cerebral malperfusion, for which the standardized differences were slightly higher (Table 1). For the matched groups, means were compared using the paired Student's $t$-test, and frequencies were compared using McNemar's test. Continuous variables not normally distributed were compared with the Wilcoxon signed-rank test.

Multivariable analyses were conducted to identify potential predictors of adverse postoperative outcomes including in-hospital mortality, postoperative stroke and paraplegia. Adverse outcomes were model by logistic regressions with stepwise selection with data from all patients $(n=646)$ by using the following preoperative and intraoperative variables: age, male, connective tissue disease, hypertension, diabetes mellitus, smoking, history of stroke, coronary heart disease, renal dysfunction, COPD, history of heart/aortic surgery, atrial fibrillation, unstable status, spinal malperfusion, cerebral malperfusion, coronary malperfusion, renal malperfusion, gastrointestinal malperfusion, iliofemoral malperfusion, supracoronary replacement, commissure resuspension, aortic root replacement, valve-sparing root replacement, total arch, subtotal arch, FET, CABG, MVP/MVR, CPB time, crossclamp time, HCA time.

Statistical analyses were performed in $\mathrm{R}$ version 3.6.0 (R Foundation, Vienna, Austria) and SPSS software v22.0 (SPSS, Inc., Chicago, IL, USA). P values $<0.05$ were considered statistically significant.

\section{Results}

The axillary artery was used in 558 procedures $(86.4 \%)$ and the femoral artery in $88(13.6 \%)$. The baseline characteristics of the two groups were different: the femoral group was more likely to have connective tissue disease, history of stroke, history of heart/aortic surgery, and incidence of renal dysfunction. After propensity score matching, the demographic characteristics of the two groups ( $\mathrm{n}=85$ in each) were comparable. The baseline characteristics for the unmatched and propensity scorematched patient populations are summarized in Table 1.

Before propensity score matching, valve-sparing root replacement was more commonly performed in the femoral group $(13.6 \%$ vs. $6.3 \%, \mathrm{P}=0.013)$, and $\mathrm{CPB}$ time was longer in the femoral group $(239.5 \pm 66.2 \mathrm{vs}$. $253.5 \pm 72.1 \mathrm{~min}, \mathrm{P}=0.033)$. More cases of total arch replacement were performed in the axillary group, but the 


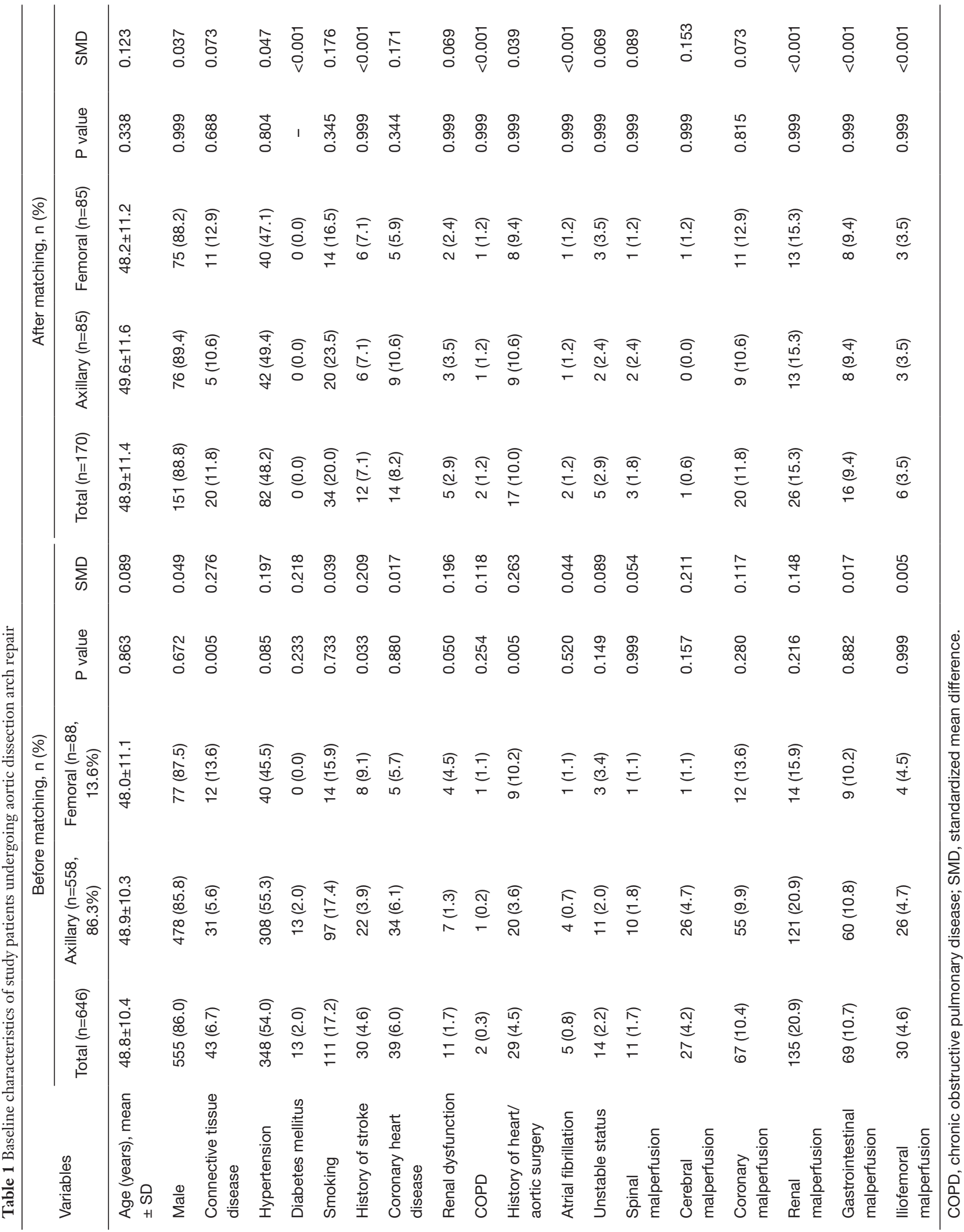


difference was not statistically significant $(89.6 \%$ vs. $83.0 \%$, $\mathrm{P}=0.067)$. In the propensity score-matched population, commissure resuspension was performed more in the femoral group, but the difference was not statistically significant (9.4\% vs. 21.2\%, $\mathrm{P}=0.064)$. More frozen elephant trunk (FET) procedures were performed in the axillary group, but the difference was not statistically significant (100\% vs. 94.1\%, $\mathrm{P}=0.063$ ). Other intraoperative variables were comparable between the two groups after matching (Table 2).

\section{Stroke}

In the entire population, early embolic stroke occurred in 35 patients $(5.4 \%)$. Before propensity score matching, the incidence of stroke was comparable between the two groups (5.4\% vs. $5.7 \%, \mathrm{P}=0.804)$. In the propensity score-matched population, the incidence of stroke was comparable between groups (3.5\% vs. $5.9 \% ; \mathrm{P}=0.687$ ) (Table 3).

\section{In-bospital mortality and early complications}

In the entire study population, 71 patients $(11.0 \%)$ died during hospital stay. Before matching, the mortality rate was $10.6 \%$ in the axillary group versus $13.6 \%$ in the femoral group $(\mathrm{P}=0.393)$. In the propensity score-matched population, in-hospital mortality was comparable between the axillary and femoral groups (10.6\% vs. $14.1 \% ; \mathrm{P}=0.648)$. The incidence of re-exploration for bleeding, ECMO use, mediastinitis and tracheostomy were comparable between groups both before and after matching. After matching, the incidence of newly required dialysis was lower in the femoral group, but the difference was not statistically significant $(34.1 \%$ vs. $20.0 \% ; \mathrm{P}=0.050)$. The length of ventilation time was comparable between groups before and after matching. However, after matching, patients in the femoral group were more likely to require a shorter stay in the intensive care unit (Table 3).

\section{Multivariable analysis for identifying risk factors of adverse outcomes}

In the multivariable analysis, aortic root replacement and renal malperfusion were identified as predictors of inhospital mortality. Cerebral malperfusion and history of stroke was predictive of postoperative stroke while spinal malperfusion and coronary malperfusion predicted increased risk of paraplegia. In agreement with results of propensity score match analysis, femoral cannulation was not predictive of any of the 3 adverse outcomes (Table 4).

\section{Discussion}

In this propensity score-matched study, we compared different cannulation strategies for open arch repair and found that femoral artery cannulation produced similar outcomes for selected patients as compared with axillary artery cannulation.

The arterial cannulation strategy is vital for outcomes in TAAD patients undergoing arch repair. Despite many improvements in treating TAAD, in-hospital mortality and complications after surgery remain high $(13,14)$. Perioperative mortality and complications are strongly associated with malperfusion and vary according to the number of organ systems affected (15). The surgeon's cannulation strategy is a critical factor that defines organ perfusion during surgery and therefore may play a crucial role in the operative outcome (16).

Theoretically, axillary artery cannulation lowers the risk of retrograde debris embolization and further dissection of the intimal flap and expansion of the false lumen that may occur with femoral cannulation and retrograde flow in the aorta. Axillary artery cannulation has been demonstrated to produce superior perioperative and long-term outcomes compared with femoral artery cannulation in patients with TAAD by multiple studies including a meta-analysis $(1,3,17,18)$. These studies lead to the 2014 European Society of Cardiology Guidelines on the Diagnosis and Treatment of Aortic Diseases recommending the axillary artery as the first choice for cannulation in surgery of the aortic arch and in type A dissection (class of recommendation I, level of evidence C) (4). In a most recent review of the Society of Thoracic Surgeons (STS) Database, axillary cannulation was associated with lower incidence of stroke, whereas femoral cannulation was found to significantly increase the risk of stroke regardless of the cerebral perfusion strategy or the degree of hypothermia (16).

However, axillary cannulation should not be used indiscriminately (5). The safety of axillary cannulation is largely dependent on the absence of extensive axillary dissection to the cannulation site or intimal tear within the innominate or subclavian artery, as false-lumen perfusion may occur, and occlusion of the right common carotid artery via true-lumen collapse by the pressurized false lumen may be particularly hazardous. Sporadical catastrophic aortic ruptures have been reported $(6,19)$. In 


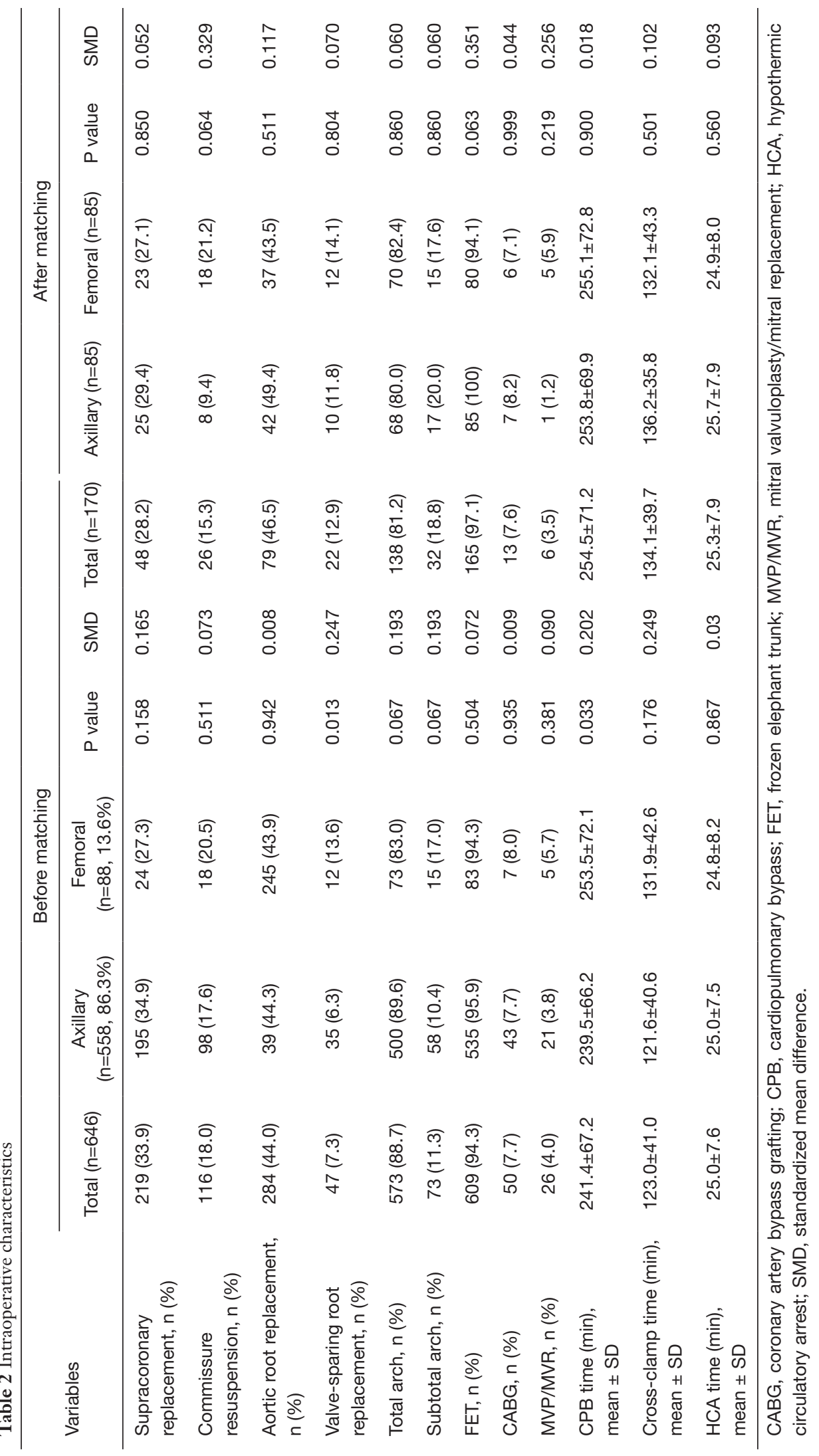




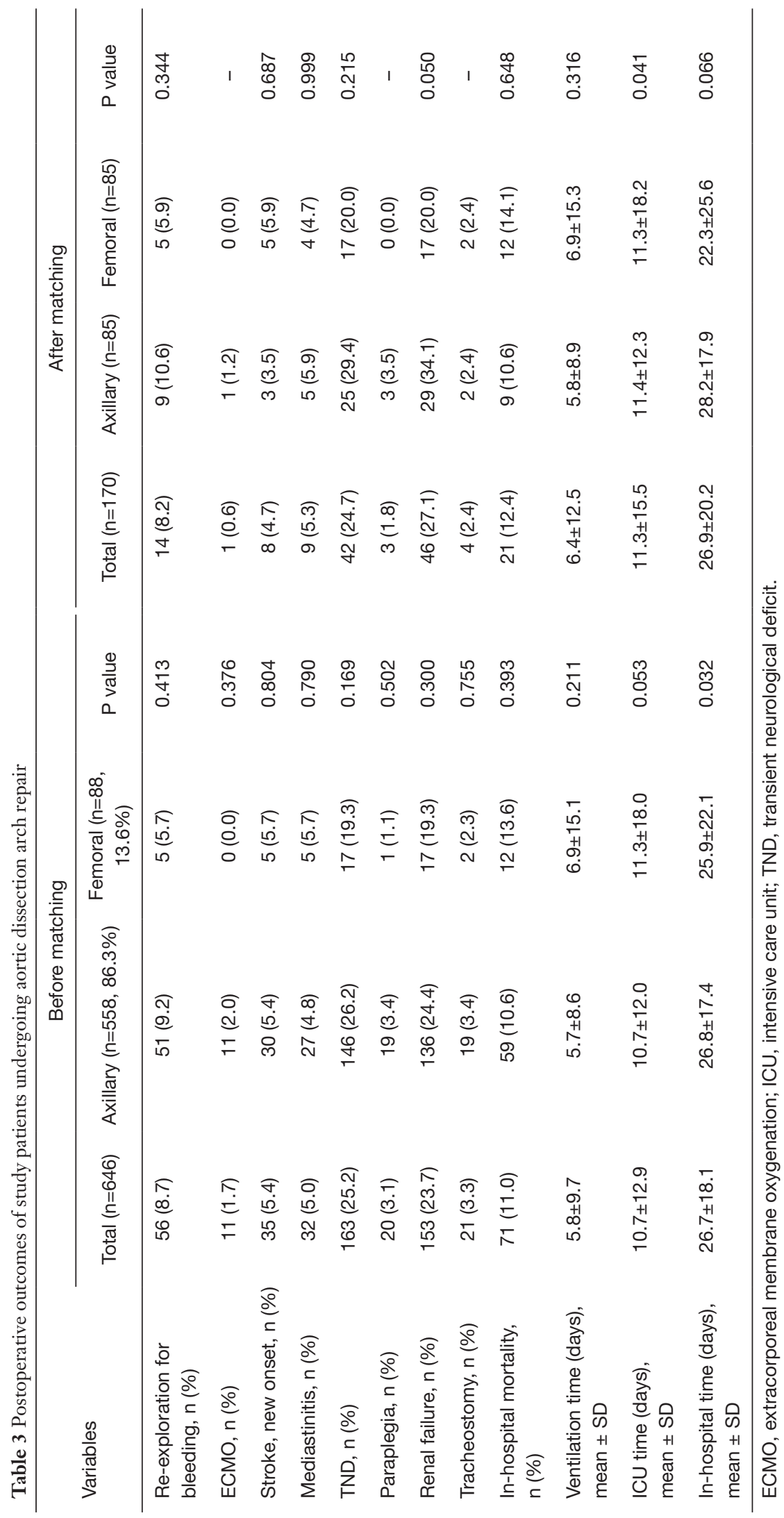


Table 4 Multivariable analysis

\begin{tabular}{|c|c|c|c|}
\hline Outcome & Predictor variables & $P$ value & Odds ratio $(95 \% \mathrm{Cl})$ \\
\hline \multirow{2}{*}{ In-hospital mortality } & Renal malperfusion & 0.048 & $1.820(1.004-3.298)$ \\
\hline & Femoral cannulation & NA & NA \\
\hline \multirow[t]{2}{*}{ Stroke, new onset } & Cerebral malperfusion & 0.024 & $3.586(1.183-10.870)$ \\
\hline & Femoral cannulation & NA & NA \\
\hline \multirow[t]{3}{*}{ Paraplegia } & Spinal malperfusion & 0.012 & $8.045(1.584-40.874)$ \\
\hline & Coronary malperfusion & 0.035 & $3.128(1.086-9.007)$ \\
\hline & Femoral cannulation & N/A & NA \\
\hline
\end{tabular}

NA, not applicable.

the present study, femoral cannulation was chosen for 47 patients because of the presence of extensive axillary artery dissection or possible intimal tear within the innominate or subclavian artery.

Not uncommonly, the axillary artery has insufficient caliber, which can lead to technical problems and complications during cannulation (6). For 29 patients in the present study, axillary cannulation was converted to femoral artery cannulation due to insufficient caliber upon surgical inspection, low blood reflows in the cannula, or high arterial resistance noted immediately after CPB initiation.

ARSA is an uncommon congenital aortic arch anomaly, with a reported incidence of $0.2-1.7 \%$ (20). It can also be involved in aortic dissection and thus influence the cannulation strategy $(5,21)$. In the presence of ARSA, right axillary artery cannulation cannot be used for ASCP, because it does not share a common origin with the right common carotid artery. If the right axillary artery is chosen for arterial cannulation, a direct carotid artery access should be added for ASCP. If its origin is blocked, ARSA should also be reconstructed by inserting a descending aorta stent to avoid right upper limb and cerebral posterior circulation ischemia (22). We avoided axillary artery cannulation in the presence of ARSA in most cases, because we usually perform FET. However, 12 patients were identified with ARSA and the femoral artery was the primary arterial cannulation site with the left common carotid artery used to directly perfuse for ASCP.

Femoral cannulation was commonly used before the axillary artery access was popularized and produced acceptable outcomes. Some of the latest studies report that femoral artery cannulation produces similar outcomes to other cannulation strategies $(23,24)$. One major concern over the use of femoral cannulation is the potential atherosclerotic embolism associated with retrograde aortic flow. In the present study, femoral artery cannulation produced a similar stroke rate to axillary artery cannulation. One most likely explanation is that the present patients were at least 10 years younger (average age: 48.8 years) than in most of the studies that did not favor femoral artery cannulation (25-27). This difference in patient demographics made atherosclerotic embolism a lesser concern.

We reported similar perioperative outcomes for the axillary and femoral cannulation strategies. Specific patient groups may benefit from certain cannulation strategies. An individualized approach should be used to choose the optimal cannulation site. Timely judgment and flexibility in the conversion of perfusion techniques are also vital for good results.

\section{Study limitations}

Because this was a retrospective study, axillary and femoral cannulation was not randomly assigned and therefore may have been subject to selection bias and uncontrolled confounding. Indications for femoral and axillary cannulation are inherently different. Propensity scorematched analysis was performed to adjust for differences in risk stratification and account for selection biases. However, as with all observational analyses, bias and confounding may persist despite our attempts to control for them. 


\section{Conclusions}

An individualized arterial cannulation approach should be used in arch repair for TAAD. In patients for whom axillary artery cannulation was considered unsuitable, femoral artery cannulation produced similar perioperative outcomes to axillary artery cannulation. Femoral cannulation is a safe alternative to axillary cannulation in aortic arch repair for TAAD patients.

\section{Acknowledgments}

We thank K. Brown and J. Gray of AME for English language editing.

Funding: This work was supported by the National Natural Science Foundation of China (81500183 to GT), the Guangdong Project of Science and Technology (2017A070701013 to TS) and the Guangzhou Project of Science and Technology (202002020023 to TS).

\section{Footnote}

Reporting Checklist: The authors have completed the STROBE reporting checklist. Available at http://dx.doi. org/10.21037/jtd-20-2113

Data Sharing Statement: Available at http://dx.doi. org/10.21037/jtd-20-2113

Conflicts of Interest: All authors have completed the ICMJE uniform disclosure form (available at http://dx.doi. org/10.21037/jtd-20-2113). The authors have no conflicts of interest to declare.

Ethical Statement: The authors are accountable for all aspects of the work in ensuring that questions related to the accuracy or integrity of any part of the work are appropriately investigated and resolved. The study protocol was approved by the Institutional Ethics Committee of Guangdong Provincial People's Hospital [approval number: No. GDREC2018322H(R2)]. Owing to the retrospective nature of the study, individual consent was waived.

Open Access Statement: This is an Open Access article distributed in accordance with the Creative Commons Attribution-NonCommercial-NoDerivs 4.0 International License (CC BY-NC-ND 4.0), which permits the noncommercial replication and distribution of the article with the strict proviso that no changes or edits are made and the original work is properly cited (including links to both the formal publication through the relevant DOI and the license). See: https://creativecommons.org/licenses/by-nc-nd/4.0/.

\section{References}

1. Ren Z, Wang Z, Hu R, et al. Which cannulation (axillary cannulation or femoral cannulation) is better for acute type A aortic dissection repair? A meta-analysis of nine clinical studies. Eur J Cardiothorac Surg 2015;47:408-15.

2. Kim JH, Lee SH, Lee S, et al. Axillary artery cannulation reduces early embolic stroke and mortality after open arch repair with circulatory arrest. J Thorac Cardiovasc Surg 2020;159:772-8.e4.

3. Benedetto U, Mohamed H, Vitulli P, et al. Axillary versus femoral arterial cannulation in type A acute aortic dissection: evidence from a meta-analysis of comparative studies and adjusted risk estimates. Eur J Cardiothorac Surg 2015;48:953-9.

4. Erbel R, Aboyans V, Boileau C, et al. 2014 ESC Guidelines on the diagnosis and treatment of aortic diseases:

Document covering acute and chronic aortic diseases of the thoracic and abdominal aorta of the adult. The Task Force for the Diagnosis and Treatment of Aortic Diseases of the European Society of Cardiology (ESC). Eur Heart J 2014;35:2873-926.

5. Maxwell BG, Harrington KB, Beygui RE, et al. Congenital anomalies of the aortic arch in acute type-a aortic dissection: implications for monitoring, perfusion strategy, and surgical repair. J Cardiothorac Vasc Anesth 2014;28:467-72.

6. Schachner T, Nagiller J, Zimmer A, et al. Technical problems and complications of axillary artery cannulation. Eur J Cardiothorac Surg 2005;27:634-7.

7. Fukuda I. Management of intraoperative malperfusion syndrome using femoral artery cannulation for repair of acute type A aortic dissection. Invited commentary. Ann Thorac Surg 2008;85:1624.

8. Ayyash B, Tranquilli M, Elefteriades JA. Femoral artery cannulation for thoracic aortic surgery: safe under transesophageal echocardiographic control. J Thorac Cardiovasc Surg 2011;142:1478-81.

9. Van Arsdell GS, David TE, Butany J. Autopsies in acute type A aortic dissection. Surgical implications. Circulation 1998;98:II299-302; discussion II302-294.

10. Kamiya H, Kallenbach K, Halmer D, et al. Comparison of 
ascending aorta versus femoral artery cannulation for acute aortic dissection type A. Circulation 2009;120:S282-6.

11. Orihashi K. Malperfusion in acute type a aortic dissection: unsolved problem. Ann Thorac Surg 2013;95:1570-6.

12. Silber JH, Rosenbaum PR, Trudeau ME, et al. Multivariate matching and bias reduction in the surgical outcomes study. Med Care 2001;39:1048-64.

13. Conzelmann LO, Weigang E, Mehlhorn U, et al. Mortality in patients with acute aortic dissection type A: analysis of pre- and intraoperative risk factors from the German Registry for Acute Aortic Dissection Type A (GERAADA). Eur J Cardiothorac Surg 2016;49:e44-52.

14. Pape LA, Awais M, Woznicki EM, et al. Presentation, Diagnosis, and Outcomes of Acute Aortic Dissection: 17Year Trends From the International Registry of Acute Aortic Dissection. J Am Coll Cardiol 2015;66:350-8.

15. Czerny M, Schoenhoff F, Etz C, et al. The Impact of PreOperative Malperfusion on Outcome in Acute Type A Aortic Dissection: Results From the GERAADA Registry. J Am Coll Cardiol 2015;65:2628-35.

16. Ghoreishi M, Sundt TM, Cameron DE, et al. Factors associated with acute stroke after type A aortic dissection repair: An analysis of the Society of Thoracic Surgeons National Adult Cardiac Surgery Database. J Thorac Cardiovasc Surg 2020;159:2143-54.e3.

17. Haldenwang PL, Wahlers T, Himmels A, et al. Evaluation of risk factors for transient neurological dysfunction and adverse outcome after repair of acute type A aortic dissection in 122 consecutive patients. Eur J Cardiothorac Surg 2012;42:e115-20.

18. Etz CD, von Aspern K, da Rocha ESJ, et al. Impact of perfusion strategy on outcome after repair for acute type a

Cite this article as: Tong G, Zhuang DL, Sun ZC, Chen ZR, Fan RX, Sun TC. Femoral artery cannulation as a safe alternative for aortic dissection arch repair in the era of axillary artery cannulation. J Thorac Dis 2021;13(2):671-680. doi: $10.21037 /$ jtd-20-2113 aortic dissection. Ann Thorac Surg 2014;97:78-85.

19. Imanaka K, Kyo S, Tanabe H, et al. Fatal intraoperative dissection of the innominate artery due to perfusion through the right axillary artery. J Thorac Cardiovasc Surg 2000;120:405-6.

20. Freed K, Low VH. The aberrant subclavian artery. AJR Am J Roentgenol 1997;168:481-4.

21. Zhu JM, Qi RD, Liu YM, et al. Repair of complicated type $\mathrm{B}$ dissection with an aberrant right subclavian artery. Interact Cardiovasc Thorac Surg 2016;22:718-22.

22. Ren CW, Lai YQ, Yang S, et al. Four-branch Prosthetic Graft Used for Stanford Type A Aortic Dissection with Aberrant Right Subclavian Artery. Chin Med J (Engl) 2015;128:1558.

23. Ram E, Krupik Y, Lipey A, et al. Is Axillary Better Than Femoral Artery Cannulation in Repair of Acute Type A Aortic Dissection? Innovations 2019;14:124-33.

24. Kreibich M, Chen Z, Rylski B, et al. Outcome after aortic, axillary, or femoral cannulation for acute type A aortic dissection. J Thorac Cardiovasc Surg 2019;158:27-34.e9.

25. Sadi L, Tonnessen T, Pillgram-Larsen J. Short and long-term survival in type A aortic dissection justifies the operative risk and effort. Scand Cardiovasc J 2012;46:45-50.

26. Reuthebuch O, Schurr U, Hellermann J, et al. Advantages of subclavian artery perfusion for repair of acute type A dissection. Eur J Cardiothorac Surg 2004;26:592-8.

27. Etz CD, Plestis KA, Kari FA, et al. Axillary cannulation significantly improves survival and neurologic outcome after atherosclerotic aneurysm repair of the aortic root and ascending aorta. Ann Thorac Surg 2008;86:441-6; discussion 446-7. 